\title{
Motional narrowing effect in certain random binary lattices
}

\author{
F. Domínguez-Adame \\ GISC, Departamento de Física de Materiales, Universidad \\ Complutense, E-28040 Madrid, Spain
}

(October 10, 2013)

\begin{abstract}
We present a model for a class of random binary lattices by introducing a one-dimensional system where impurities are placed in one sublattice while host atoms lie on the other sublattice. The source of disorder is the stochastic fluctuation of the impurity energy from site to site. We study the optical absorption spectra and the peculiarities of the motional narrowing effect at the band edges for perturbative and nonperturbative degrees of disorder. Analytical results agree well with numerical simulations.
\end{abstract}

\section{INTRODUCTION}

Several years ago, Knapp raised the concept of motional or exchange narrowing in one-dimensional (1D) disordered systems [1] and fruitfully explained many optical phenomena in low-dimensional disordered systems like J aggregates and conjugated polymers (for a review, see Refs. [2,3] and references therein). Roughly speaking, this author demonstrated that the disorder seen by the quasi-particles of the excited system is reduced as compared to the seeding degree of disorder, namely the width of the probability distribution of the on-site energy, as soon as the states of the individual molecules are collectivized due to the intermolecular interactions. The suppression factor depends on whether or not the disorder is small enough to be regarded as a perturbation. In the perturbative case, this factor is determined by the square root of the number of sites in the entire system, while in the nonperturbative case this number should be substituted by the so-called number of coherently bound molecules, due to the occurrence of the Anderson localization whenever the on-site energy is an uncorrelated random variable.

Numerically simulated absorption spectrum of polysilane with an uncorrelated Gaussian distribution of nearest-neighbor couplings are similar to those for an uncorrelated diagonal disorder [4]. In contrast, simulations of off-diagonal disorder given by Gaussian randomness in the molecular positions [5] found that the behavior of the optical observables does not fit the standard motional narrowing effect [1]. Recently, this discrepancy has been uncovered by Malyshev and Domínguez-Adame [6], pointing out the appearance of correlations in the intersite couplings even if the fluctuations of the molecular positions are uncorrelated. Furthermore, it is nowadays well established both theoretically [7,8] and experimentally [9] that extended states may arise in $1 \mathrm{D}$ random systems when disorder is correlated, in contrast to the earlier believe that all eigenstates should be localized [10]. The motional narrowing effect and the competition between the localization length and the correlation length have been considered very recently by Rodríguez et al. [11.

In this work, we report further progress along the lines in the preceding paragraphs. In particular, we focus our attention on a new 1D tight-binding model of correlated disordered system supporting extended states [12,13]. We built up our model by considering a $1 \mathrm{D}$ random binary lattice with two species, referred to as $\mathrm{A}$ and $\mathrm{B}$ atoms hereafter. We further assume that the site energy of A atoms is randomly distributed from site to site while that of B atoms is the same over the other sublattice. We have demonstrated analytically the occurrence of extended states in the vicinity of the site energy of B atoms in spite of the fact that the system is purely $1 D$ and random [13]. Here we study the peculiarities of the motional narrowing effect and their manifestation through the optical properties of this system.

\section{MODEL}

Let us consider a tight-binding Hamiltonian with nearest-neighbor interactions

$$
\mathcal{H}=\sum_{n}\left[\epsilon_{n}|n\rangle\langle n|-J| n\rangle\langle n+1|-J| n+1\rangle\langle n|\right],
$$

where the state vector $|n\rangle$ represents an excitation at site $n$. In the present 1D binary system, A (B) atoms are placed at odd (even) positions of the otherwise regular lattice, whose corresponding site energies are $\epsilon_{2 n-1}\left(\epsilon_{2 n}\right)$ with $n=1,2, \ldots N, N$ being the number of unit cells of the system. According to our model, site energies at even positions are the same and we can set $\epsilon_{2 n}=0$ without loss of generality. The source of disorder arise from the stochastic fluctuations of site energy at odd positions. We assume that $\left\{\epsilon_{2 n-1}\right\}_{n=1}^{N}$ is a set of uncorrelated random Gaussian variables with mean value $v$ and variance $\sigma^{2}$. Hereafter $\sigma$ will be referred to as degree of disorder. The joint distribution function is represented by the direct product of single Gaussians. Thus 


$$
\left\langle\epsilon_{2 n-1}\right\rangle_{\mathrm{av}}=v, \quad\left\langle\epsilon_{2 n-1} \epsilon_{2 n^{\prime}-1}\right\rangle_{\mathrm{av}}=\left(v^{2}+\sigma^{2}\right) \delta_{n n^{\prime}},
$$

where the angular brackets $\langle\ldots\rangle_{\text {av }}$ indicate the average over the ensemble. Although the system is onedimensional and random, it has been demonstrated analytically the existence of a delocalized state in infinite systems at $E=0$ [13]. Most important, there exist many $(\sim \sqrt{N})$ states close to the resonant energy that remain extended in finite systems, in the sense that their localization length is larger than the system size.

\section{PERTURBATIVE MOTIONAL NARROWING EFFECT}

We now calculate the motional narrowing effect in the perturbative limit by considering small degree of disorder $\sigma$. To this end, we start by writing down the eigenstates and eigenenergies of the unperturbed Hamiltonian $(\sigma=0)$, corresponding to a diatomic lattice with onsite energies 0 and $v$ in each unit cell. We assume rigid boundary conditions and $v>0$. The last restriction is only for the sake of clarity in the exposition of results, as they are trivially generalized to the case $v<0$. There are two allowed bands, the first one ranging from $v / 2-\sqrt{(v / 2)^{2}+4 J^{2}}$ up to 0 and the second one ranging from $v$ up to $v / 2+\sqrt{(v / 2)^{2}+4 J^{2}}$ for $v>0$. The normalized eigenstates of the unperturbed Hamiltonian can be written as

$$
\begin{aligned}
|K, \pm\rangle & =\sqrt{\frac{4}{2 N+1}} \sum_{n=1}^{N}\left\{\alpha_{K}^{ \pm} \sin [(2 n-1) K]|2 n-1\rangle\right. \\
& \left.+\beta_{K}^{ \pm} \sin (2 n K)|2 n\rangle\right\}
\end{aligned}
$$

where $K \equiv k \pi /(2 N+1)$ with $k=1,2, \ldots, N$ and

$$
\begin{aligned}
& \alpha_{K}^{ \pm}=\left[1+\frac{E_{K}^{ \pm}}{E_{K}^{ \pm}-v}\right]^{-1 / 2}, \\
& \beta_{K}^{ \pm}=\left[1+\frac{E_{K}^{ \pm}-v}{E_{K}^{ \pm}}\right]^{-1 / 2} .
\end{aligned}
$$

Here the labels \pm refer to the upper $(+)$ and the lower $(-)$ band, respectively. The dispersion relation within each band is given by

$$
E_{K}^{ \pm}=\frac{v}{2} \pm \sqrt{\frac{v^{2}}{4}+4 J^{2} \cos ^{2} K} .
$$

The perturbative part of the Hamiltonian, $\mathcal{H}_{p}$, is diagonal in the site representation and comes from the stochastic fluctuations of the on-site energy at odd sites around the mean value $v$. Therefore $\mathcal{H}_{p}=\sum_{n=1}^{N}\left(\epsilon_{2 n-1}-\right.$ $v)|2 n-1\rangle\langle 2 n-1|$. In the $K$-representation (35), the matrix elements of $\mathcal{H}_{p}$ are expressed through linear combinations of Gaussian variables $\epsilon_{2 n-1}-v$ with zero mean

$$
\begin{aligned}
& \left\langle K, \ell\left|\mathcal{H}_{p}\right| K^{\prime}, \ell^{\prime}\right\rangle=\frac{4 \alpha_{K}^{\ell} \alpha_{K^{\prime}}^{\ell^{\prime}}}{2 N+1} \times \\
& \sum_{n=1}^{N}\left(\epsilon_{2 n-1}-v\right) \sin [(2 n-1) K] \sin \left[(2 n-1) K^{\prime}\right],
\end{aligned}
$$

where $\ell$ and $\ell^{\prime}$ indicate the band. Consequently, they also have a joint Gaussian distribution with zero mean. In what follows, we will deal with the fluctuations of the square of the matrix elements given in (6) since they determine the linear optical response of the system.

Concerning optical transitions, since the states with $k=1$ (one per band) carry almost the entire oscillator strength of the system, in the perturbative limit the optical absorption spectrum is dominated by two Gaussian peaks located at the bottom (top) of the lower (upper) band. It is easy to demonstrate that the oscillator strength is proportional to $\alpha_{K}^{ \pm} \mp \beta_{K}^{ \pm}$, where $\alpha_{K}^{ \pm}$and $\beta_{K}^{ \pm}$are given by (身), so that the lower energy transition becomes more intense. Therefore, we restrict ourselves to this transition, whose standard deviation is given by $\sigma_{1}=B_{11}$ with $B_{k k^{\prime}}^{2} \equiv\left\langle\left\langle K,-\left|\mathcal{H}_{p}\right| K^{\prime},-\right\rangle^{2}\right\rangle_{\mathrm{av}}$. After performing the summations (6) one can obtain $B_{k k^{\prime}}$. Close to the zone center, which is responsible of the linear optical response of the system, the calculation yields

$$
B_{k k^{\prime}}=\frac{\sqrt{v^{2}+16 J^{2}}-v}{2 \sqrt{v^{2}+16 J^{2}}} \sqrt{\frac{2+\delta_{k k^{\prime}}}{2 N+1}} \sigma, \quad k, k^{\prime} \ll N,
$$

where in (4) we have replaced $E_{K}^{-}$and $E_{K^{\prime}}^{-}$by their values at the zone center since $K \rightarrow 0$. Therefore, the standard deviation of the main absorption line is given by

$$
\sigma_{1}=\frac{\sqrt{v^{2}+16 J^{2}}-v}{2 \sqrt{v^{2}+16 J^{2}}} \sqrt{\frac{3}{2 N+1}} \sigma .
$$

As it can be seen from (8), the standard deviation of the Gaussian peak scales as $N^{-1 / 2}$, showing the so-called motional narrowing effect [1]. Notice that the reduction factor carries information on the system parameter $v / J$.

\section{NONPERTURBATIVE DEGREE OF DISORDER}

The perturbative approach we have carried out in the preceding section holds for small degree of disorder. On increasing the degree of disorder, mixing of the states $k=$ 1 with the other states strongly affect the system optical response. Hence, one should compare the energy difference between the two lowest states, $\delta E_{12} \equiv\left|E_{K_{1}}^{-}-E_{K_{2}}^{-}\right|$, with the typical fluctuation of $\left\langle K_{1},-\left|\mathcal{H}_{p}\right| K_{2},-\right\rangle$ represented by $B_{12}$, where $K_{m} \equiv m \pi /(2 N+1)$. The perturbative approach is valid provided $\delta E_{12}>B_{12}$ and fails otherwise. The energy difference can be readily determined from (5) 


$$
\delta E_{12}=\frac{12 \pi^{2} J^{2}}{\sqrt{v^{2}+16 J^{2}}} \frac{1}{(2 N+1)^{2}}, \quad N \gg 1 .
$$

For higher values of the degree of disorder $\left(\delta E_{12}<B_{12}\right)$ the perturbative approach fails; not all sites contribute to the optical spectrum since the Anderson localization length becomes smaller than the system size. Thus, $N$ should be replaced by $N^{*}<N$ in the above equations [1], where $N^{*}$ is often referred to as the number of coherently bound molecules. Malyshev proposed a selfconsistent estimation of $N^{*}$ by applying the perturbative criterion mentioned above to a typical localization segment of length $N^{*} \sqrt[14]{14}$. This approach yields excellent results for uncorrelated and pairwise correlated diagonal disorder [15]. Thus, we use the condition $B_{12}^{*}=\delta E_{12}^{*}$, where the asterisk means the substitution of $N$ by $N^{*}$ in (7) and (9). In doing so we obtain

$$
\frac{1}{\sqrt{2 N^{*}+1}}=\left(\frac{\sqrt{v^{2}+16 J^{2}}-v}{12 \sqrt{2} \pi^{2} J^{2}} \sigma\right)^{1 / 3} .
$$

Finally, replacing in (8) $N$ by $N^{*}$ given by (10) we get for the standard deviation of the peaks

$$
\sigma_{1}^{*}=C \frac{\sigma^{4 / 3}}{J^{1 / 3}}
$$

where

$$
C=\frac{\sqrt{3}}{2\left(12 \sqrt{2} \pi^{2}\right)^{1 / 3}} \frac{\left[\sqrt{(v / J)^{2}+16}-v / J\right]^{4 / 3}}{\sqrt{(v / J)^{2}+16}} .
$$

This expression holds for $v>0$ as well as for $v<0$. The power-like dependence $\sigma^{4 / 3}$ also appears in dealing with uncorrelated and pairwise correlated diagonal disorder but with different $C$ (see, e.g., Ref [15]) and can be obtained from a more rigorous framework using the coherent potential approximation 16.

\section{NUMERICAL SIMULATIONS}

To check the accuracy of the above analytical approach, we have obtained numerically the absorption line shape, $I(E)$, according to Ref. [5]. We fix the value $J=1$ and focus our attention on the degree of disorder $\sigma$, ranging from 0.1 up to 0.9. We have diagonalized the Hamiltonian (11) for chains of $N=125$ (250 sites) with rigid boundary conditions. The number of randomly generated systems is 10000 for each value of $\sigma$. We show in Fig. 1 several examples of the optical absorption spectra for various values of the parameters $v$ and $\sigma$; only the lower energy side of the spectra is shown. For small values of $\sigma$ the position of the main line agrees very well with the value $v / 2-\sqrt{(v / 2)^{2}+4 J^{2}}$ obtained from (5). This main line broadens and becomes redshifted on increasing the degree of disorder, as expected.

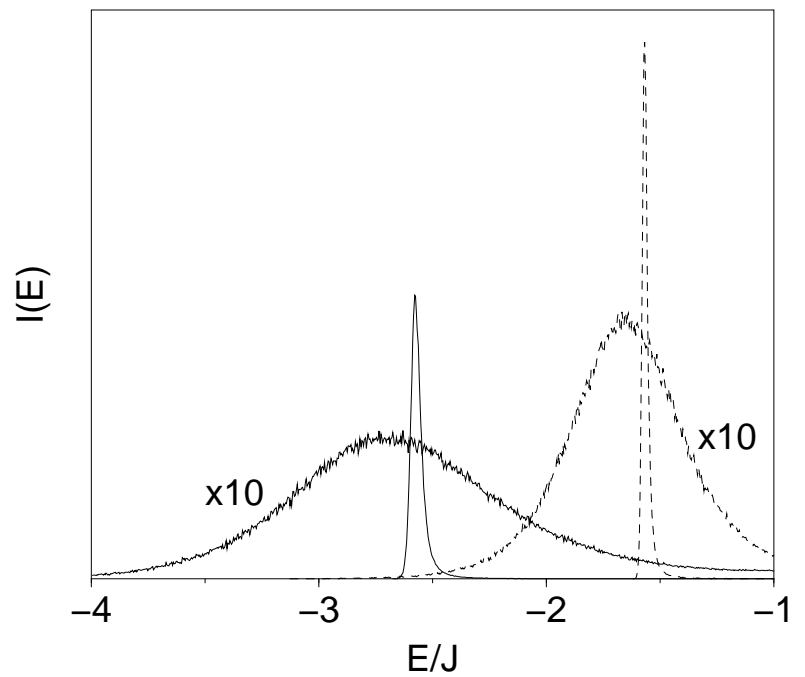

FIG. 1. Absorption spectra for one-dimensional random binary lattices with $J=1, N=125$ (250 sites) and $v=-1$ (solid lines) and $v=+1$ (dashed lines). The narrow and broad peaks correspond to $\sigma=0.1$ and $\sigma=0.9$, respectively. Notice the scale factor in the later case. Each spectrum comprises the results of 10000 realizations of the disordered system.

The value of $\sigma_{1}^{*}$ was obtained by nonlinear Gaussian fitting of the low energy side of the spectra and the results are presented in Fig. 2. It is found that this magnitude scales as $\sigma^{4 / 3}$ for the various values of $v$ studied, as predicted by our estimates (11a). The slopes of the straight lines are slightly larger than those obtained from (11b), as shown in Table I; nevertheless, the coincidence should be admitted as being highly surprising in view of the simple assumptions we made.

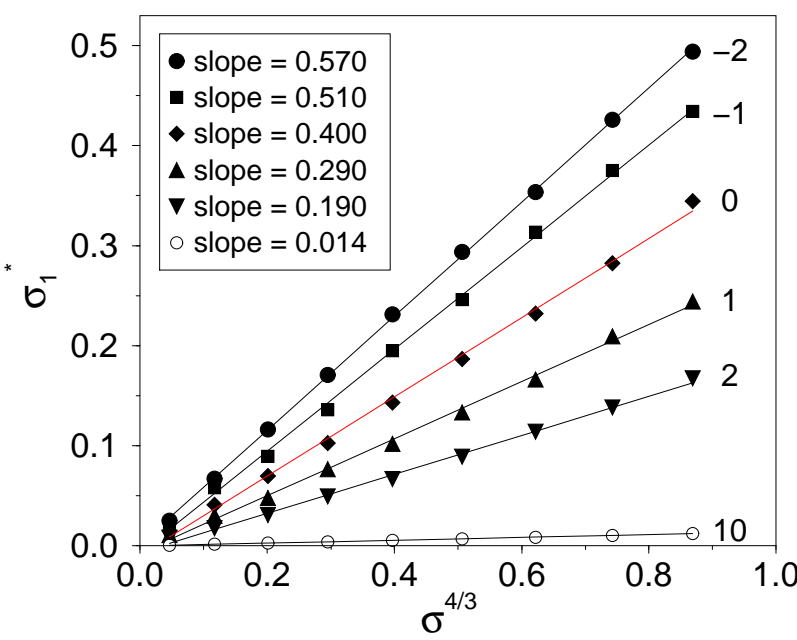

FIG. 2. Standard deviation $\sigma_{1}^{*}$ as a function of $\sigma^{4 / 3}$ for different values of $v$, indicated at the right of each line, with $J=1$ and $N=125$ (250 sites). Solid lines represent the least square fits and each point comprises the results of 10000 realizations of the disordered system. 


\section{CONCLUSIONS}

In this paper we have considered the motional narrowing effect in a $1 \mathrm{D}$ random binary lattice where disorder lies in one of the two sublattices. This system could be regarded as a simple model of semiconductor binary alloy - like ternary III-V compounds-. In these alloys (say $\mathrm{Al}_{x} \mathrm{Ga}_{1-x} \mathrm{As}$ ), the cation sublattice is occupied by the same atoms (say As) while anions (say Al and Ga) are randomly distributed over the other sublattice. Starting from a perturbative approach, we found that the width of the absorption line scales as $\sim N^{-1 / 2}$ for small degree of disorder, showing the motional narrowing effect. For larger degrees of disorder, we determined self-consistently the spatial extend of the excitation wave function, according to the prescription raised by Malyshev [14. The spatial extend of the excitation wave function is smaller than the system size and the width of the absorption line scales as $\sim \sigma^{4 / 3}$. Our estimates agree well with numerically simulated optical absorption spectra.

\section{ACKNOWLEDGMENTS}

The author thanks V. Malyshev and A. Rodríguez for helpful discussions. This work is supported by Comunidad de Madrid under Project 07N/0034/98.

[1] E. W. Knapp, Chem. Phys. 85, 73 (1984).

[2] F. C. Spano and J. Knoester, in Advances in Magnetic and Optical Resonance, Vol. 18, ed. W. S. Warren (Academic, New York, 1994), p. 117.

[3] J. Knoester and F. C. Spano, in $J$ aggregates, ed. T. Kobayashi (World Scientific, Singapur, 1996), p. 111.

[4] A. Tilgner, H. P. Trommsdorff, J. M. Zeigler, and R. M. Hochstrasser, J. Lumin. 45, 373 (1990).

[5] H. Fidder, J. Knoester, and D. A. Wiersma, J. Chem. Phys. 95, 7880 (1991).

[6] V. A. Malyshev and F. Domínguez-Adame, Chem. Phys. Lett. 313, 255 (1999).

[7] J. C. Flores, J. Phys. Condens. Matter 1 (1989) 8471.

[8] D. H. Dunlap, H.-L. Wu, and P. Phillips, Phys. Rev. Lett. 65 (1990) 88.

[9] V. Bellani, E. Diez, R. Hey, L. Toni, L. Tarricone, G. B. Parravicini, F. Domínguez-Adame, and R. GómezAlcalá, Phys. Rev. Lett. 82 (1999) 2159.

[10] P. A. Lee and T. V. Ramakrishnan, Rev. Mod. Phys. 57, 287 (1985).

[11] A. Rodríguez, V. A. Malyshev, and F. DomínguezAdame, Phys. Rev. B 60 (1999), 14140.

[12] T. Hakobyan, D. Sedrakyan, A. Sedrakyan, I. Gómez, and F. Domínguez-Adame, Phys. Rev. B 61 (2000), 11432 .
[13] F. Domínguez-Adame, I. Gómez, A. Avakyan, D. Sedrakyan, and A. Sedrakyan, phys. stat. sol. (b) (accepted for publication).

[14] V. A. Malyshev, Opt. Spectrosc. 71, 505 (1991); J. Lumin. 55, 225 (1993).

[15] F. Domínguez-Adame and V. A. Malyshev, J. Lumin. 83-84, 61 (1999).

[16] A. Boukahil and D. L. Huber, J. Lumin. 45, 13 (1990).

\begin{tabular}{|c|r|r|r|r|r|r|}
\hline \hline$v / J$ & -2 & -1 & 0 & 1 & 2 & 10 \\
\hline \hline Analytical & 0.42 & 0.34 & 0.25 & 0.17 & 0.12 & 0.010 \\
\hline Numerical & 0.57 & 0.51 & 0.40 & 0.29 & 0.19 & 0.014 \\
\hline \hline
\end{tabular}

TABLE I. Slope of $\sigma_{1}^{*}-\sigma^{4 / 3}$ plots obtained analytically from $11 \mathrm{~b}$ and numerically for different values of the parameter $v$. 